\section{Viskosität des flüssigen Systems Chlorbenzol + Brombenzol}

\section{R. Haase, M. Lethen und K.-H. Dücker}

Lehrstuhl für Physikalische Chemie II der Rheinisch-Westfälischen Technischen Hochschule Aachen

(Z. Naturforsch. 30 a, 916-917 [1975] ; eingegangen am 7. Mai 1975)

Viscosity of the Liquid System Chlorobenzene+ Bromobenzene

Results of measurements of the viscosity and of the molar volume for the ideal liquid system chlorobenzene+bromobenzene are presented. They cover the whole composition range between $0^{\circ} \mathrm{C}$ and $80^{\circ} \mathrm{C}$. Both the composition and the temperature dependence of the viscosity are discussed.

Das flüssige System Chlorbenzol + Brombenzol stellt innerhalb der Meßgenauigkeit eine ideale Mischung dar. Daher sollte die Viskosität - wie andere Transportgrößen auch - einen besonders einfachen Konzentrationsverlauf zeigen. Wir haben deshalb die Viskosität im gesamten Mischungsbereich (für die reinen Komponenten und bei neun verschiedenen Zusammensetzungen der Mischungen) bei Temperaturen zwischen $0{ }^{\circ} \mathrm{C}$ und $80^{\circ} \mathrm{C}$ (im $\mathrm{Ab}$ stand von $10^{\circ} \mathrm{C}$ und zusätzlich bei $25^{\circ} \mathrm{C}$ ) ermittelt. Darüber hinaus haben wir in demselben Konzentrations- und Temperaturbereich die Dichte und daraus das molare Volumen bestimmt.

Wie an anderer Stelle ${ }^{1}$ ausführlich dargelegt, sind die Viskositätsmessungen mit Hilfe eines Ubbelohde-Viskosimeters, die Dichtemessungen pyknometrisch durchgeführt worden. Soweit Literaturwerte vorhanden, stimmen sie recht gut mit unseren Werten überein.

Das molare Volumen $\bar{V}$ ist, wie bei einer idealen Mischung zu erwarten, bei allen Temperaturen innerhalb der Meßgenauigkeit eine lineare Funktion des Molenbruches $x_{1}$ bzw. $x_{2}$ des Chlorbenzols bzw. Brombenzols :

$$
\bar{V}=x_{1} V_{1}^{\cdot}+x_{2} V_{2}^{\cdot}=V_{1}^{\cdot}+\left(V_{2}^{\cdot}-V_{1}^{*}\right) x_{2} .
$$

Hierin bedeutet $V_{i}$ das molare Volumen der reinen flüssigen Komponente $i$. Nach Gl. (1) genügt es, wenn wir unsere experimentellen Werte für $V_{1}$ und $V_{2}$ angeben (Tabelle 1 ).

Die Viskositäten $\eta_{1}$ und $\eta_{2}$ des reinen Chlorbenzols und reinen Brombenzols findet man in Tabelle 1, die Viskosität $\eta$ der Mischung in Abhängigkeit von der Zusammensetzung bei den verschiedenen Temperaturen in Abbildung 1. (Die SI-Einheit für die Viskosität ist $\mathrm{Pa} \mathrm{s}$, die übliche und hier benutzte Einheit $\mathrm{cP}=10^{-3} \mathrm{~Pa} \mathrm{~s}$.)

Interessanterweise gilt für die Viskosität $\eta$ kein zu Gl. (1) analoger Zusammenhang. Auch ein anderer Ansatz, der sich in der Literatur findet und eine
Tab. 1. Molares Volumen $V_{1}^{*}$ bzw. $V_{2}^{*}$ und Viskosität $\eta_{1}$ bzw. $\eta_{2}$ des reinen flüssigen Chlorbenzols bzw. Brombenzols bei verschiedenen Temperaturen.

\begin{tabular}{|c|c|c|c|c|}
\hline Temperatur & $\frac{V_{1}^{\cdot}}{\mathrm{cm}^{3} \mathrm{~mol}^{-1}}$ & $\frac{V_{2}^{\cdot}}{\mathrm{cm}^{3} \mathrm{~mol}^{-1}}$ & $\frac{\eta_{1}}{\mathrm{cP}}$ & $\frac{\eta_{2}}{\mathrm{cP}}$ \\
\hline $0^{\circ} \mathrm{C}$ & 99,82 & 103,14 & 1,0610 & 1,5364 \\
\hline $10^{\circ} \mathrm{C}$ & 100,78 & 104,07 & 0,9166 & 1,3109 \\
\hline $20^{\circ} \mathrm{C}$ & 101,77 & 105,02 & 0,8030 & 1,1355 \\
\hline $25^{\circ} \mathrm{C}$ & 102,26 & 105,49 & 0,7550 & 1,0627 \\
\hline $30^{\circ} \mathrm{C}$ & 102,77 & 105,98 & 0,7118 & 0,9974 \\
\hline $40^{\circ} \mathrm{C}$ & 103,78 & 106,95 & 0,6365 & 0,8855 \\
\hline $50^{\circ} \mathrm{C}$ & 104,83 & 107,95 & 0,5724 & 0,7917 \\
\hline $60^{\circ} \mathrm{C}$ & 105,89 & 108,97 & 0,5183 & 0,7137 \\
\hline $70^{\circ} \mathrm{C}$ & 106,98 & 110,01 & 0,4712 & 0,6455 \\
\hline $80^{\circ} \mathrm{C}$ & 108,10 & 111,08 & 0,4314 & 0,5895 \\
\hline
\end{tabular}

lineare Abhängigkeit der Fluidität $(1 / \eta)$ von den Molenbrüchen fordert, trifft für unser System nicht zu. Vielmehr lassen sich unsere experimentellen Daten innerhalb der Meßgenauigkeit durch den Ansatz von Arrhenius (1887)

$$
\eta=\eta_{1}^{x_{1}} \eta_{2}^{x_{2}}
$$

beschreiben. Dies ist in Abb. 2 für $25^{\circ} \mathrm{C}$ dargestellt.

Die Abhängigkeit der Viskosität $\eta$ von der thermodynamischen Temperatur $T$ wird bei unserem System durch den Ansatz von Reynolds (1886)

$$
\eta=C \exp \{E / R T\}
$$

recht gut wiedergegeben ${ }^{1}$. Dabei ist $E$ bzw. $R$ die Aktivierungsenergie der Mischung bzw. die Gaskonstante. Die Größen $C$ und $E$ sind nur Funktionen der Zusammensetzung.

Will man auch die Abhängigkeit der Viskosität von der Zusammensetzung durch Gl. (3) korrekt beschreiben, so muß man ansetzen:

$$
C=C_{1}{ }_{1} C_{2}{ }^{x_{2}}, \quad E=E_{1} x_{1}+E_{2} x_{2},
$$

worin $E_{1}$ bzw. $E_{2}$ die Aktivierungsenergie des reinen Chlorbenzols bzw. reinen Brombenzols bedeutet. Aus den Gln. (3) und (4) folgt:

$\eta_{1}=C_{1} \exp \left\{E_{1} / R T\right\}, \quad \eta_{2}=C_{2} \exp \left\{E_{2} / R T\right\}$.

Hieraus ergibt sich mit Gl. (3) und (4) wiederum der Ansatz (2).

Für unser System gilt:

$$
\begin{array}{ll}
C_{1}=2,09 \cdot 10^{-2} \mathrm{cP}, & C_{2}=2,41 \cdot 10^{-2} \mathrm{cP}, \\
E_{1}=8,88 \cdot 10^{3} \mathrm{~J} / \mathrm{mol}, & E_{2}=9,38 \cdot 10^{3} \mathrm{~J} / \mathrm{mol} .
\end{array}
$$

Die Viskosität des flüssigen Systems Chlorbenzol + Brombenzol läßt sich also für jede Zusammensetzung und jede Temperatur zwischen $0{ }^{\circ} \mathrm{C}$ und $80^{\circ} \mathrm{C}$ mit guter Näherung nach Gl. (3) und (4) mit Hilfe der vier angegebenen Konstanten berechnen. 


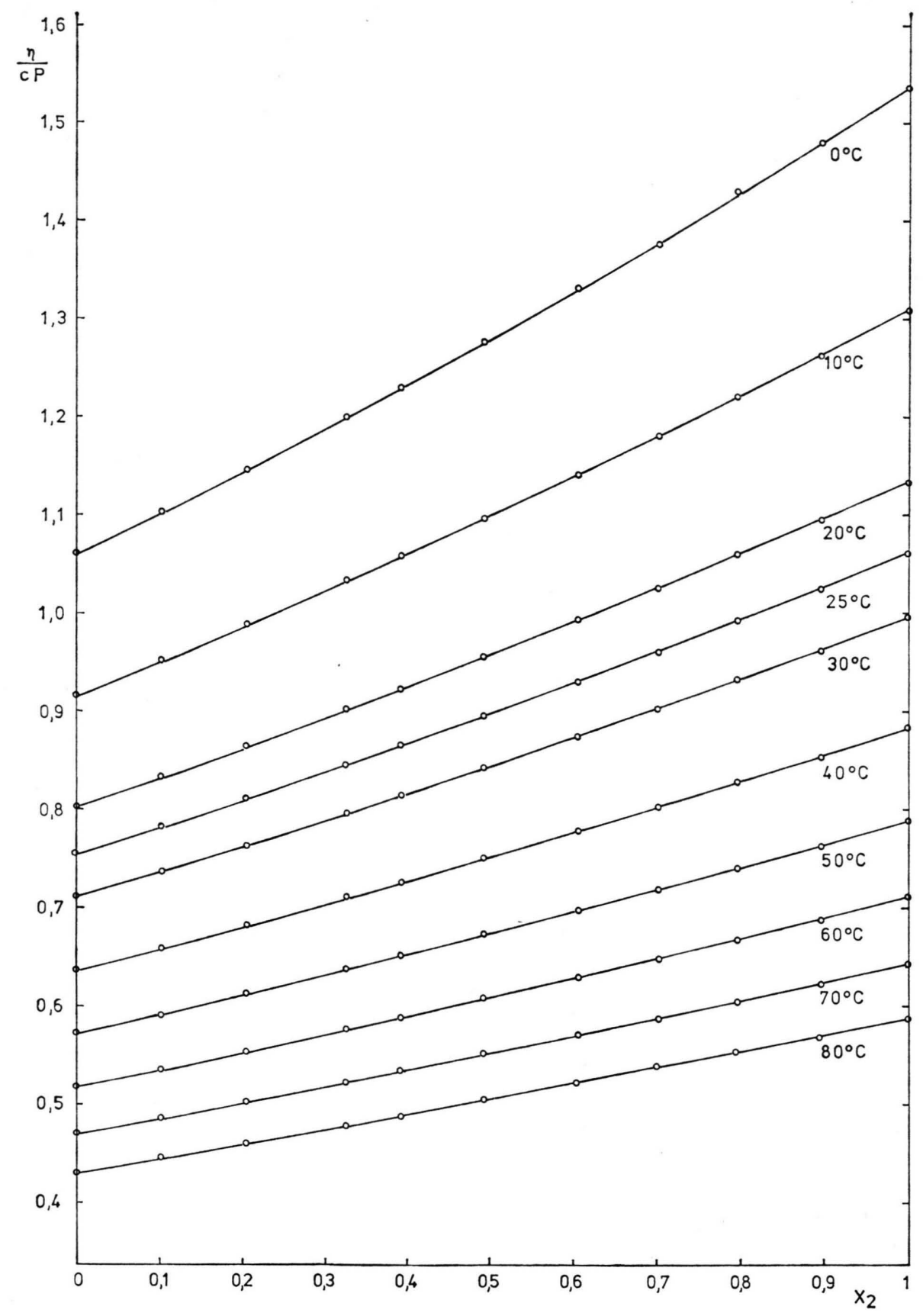

Abb. 1. Viskosität $\eta$ des flüssigen Systems Chlorbenzol+ Brombenzol in Abhängigkeit vom Molenbruch $x_{2}$ des Brombenzols bei verschiedenen Temperaturen. $\circ \mathrm{Me} \beta$ werte.

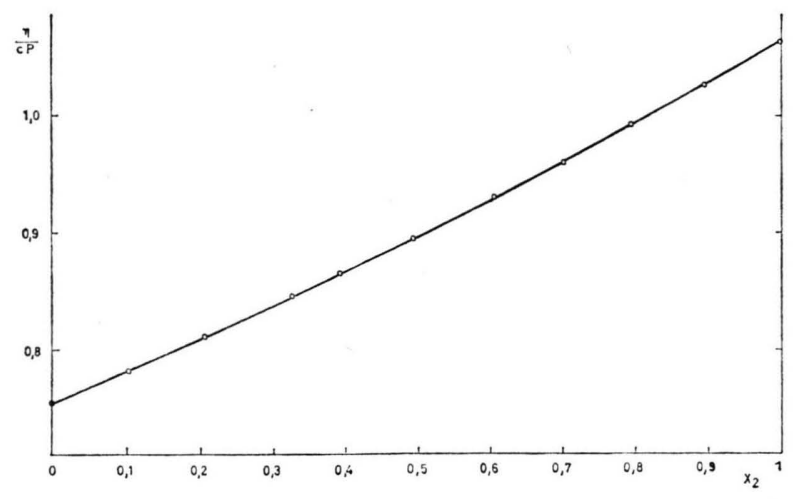

Abb. 2. Gemessene (o) und nach Gl. (2) berechnete (-) Werte der Viskosität $\eta$ des flüssigen Systems Chlorbenzol+ Brombenzol in Abhängigkeit vom Molenbruch $x_{2}$ des Brombenzols bei $25^{\circ} \mathrm{C}$.

1 M. Lethen, Die Viskosität des flüssigen Systems Chlorbenzol+Brombenzol, Diplomarbeit, RWTH Aachen 1974. 